\title{
Tailored internet-administered treatment of anxiety disorders for primary care patients: study protocol for a randomised controlled trial
}

\author{
Lise Bergman Nordgren ${ }^{1 *}$, Gerhard Andersson ${ }^{1,2,3}$, Åsa Kadowaki ${ }^{4}$ and Per Carlbring ${ }^{5}$
}

\begin{abstract}
Background: Internet-administered cognitive behavioural therapy (ICBT) has been found to be effective for a range of anxiety disorders. However, most studies have focused on one specific primary diagnosis and comorbidity has not been considered. In primary care settings, patients with anxiety often suffer from more than one psychiatric condition, making it difficult to disseminate ICBT for specific conditions. The aim of this study will be to investigate if ICBT tailored according to symptom profile can be a feasible treatment for primary care patients with anxiety disorders. It is a randomised controlled trial aimed to evaluate the treatment against an active control group.
\end{abstract}

Methods: Participants with anxiety disorders and co-morbid conditions $(N=128)$, will be recruited from a primary care population. The Clinical Outcome in Routine Evaluation (CORE-OM) will serve as the primary outcome measure. Secondary measures include self-reported depression, anxiety, quality of life and loss of production and the use of health care. All assessments will be collected via the Internet and measure points will be baseline, post treatment and 12 months post treatment.

Discussion: This trial will add to the body of knowledge on the effectiveness of ICBT for anxiety disorders in primary care. The trial will also add knowledge on the long term effects of ICBT when delivered for regular clinic patients

Trial Registration: ClinicalTrials.gov: NCT01390168

Keywords: Primary care, anxiety, depression, co-morbidity, Internet-administered cognitive behaviour therapy, cognitive behaviour therapy

\section{Background}

Anxiety symptoms are one of the most common complaints in mental health care [1]. In Europe, the 12month prevalence for any anxiety disorder is nearly 14 per cent $[2,3]$. When broadening the term to mental disorder, the number of people affected in the European Union is 23.5 per cent [3], and co-morbidity rates are high. The most common co-morbid disorder for people with one anxiety disorder is mood disorders and other anxiety disorders [4]. Although the number of people suffering from mental disorders is high, only a small

\footnotetext{
* Correspondence: lise.bergman.nordgren@liu.se

'Department of Behavioural Sciences and Learning, Linköping University, 581 83 Linköping, Sweden

Full list of author information is available at the end of the article
}

number receive treatment [5], and a large number of adult patients with anxiety disorders are treated in primary care settings and not in specialised care [6].

In an attempt to lower the barriers for help, Internetbased cognitive behavioural therapy (ICBT) has been shown to be effective [7]. ICBT can be, defined as:

"A therapy that is based on self-help books, guided by an identified therapist, which gives feedback and answers to questions, with a scheduling that mirrors face-to-face treatment, and which can also include interactive online features such as queries to obtain passwords in order to get access to treatment modules" ([7] p. 164).

This approach is different from pure self-help, in which no feedback is given from the clinician. Including

\section{Ciomed Central}


guidance has been shown to be important in order to optimise outcomes [8-10]. ICBT has shown promising results for a range of somatic and psychiatric conditions. Research has shown similar outcomes as in face-to-face therapy [11]. Moreover, there are several meta-analysis showing promising results for anxiety disorders as well as depression [10,12-14]. However, Coull and Morris [15] found that even if guided self-help seems to have a positive immediate effect, effectiveness at long-term follow-up is considerably diminished. Furthermore, the effects among clinically representative samples are largely unknown. In addition, most studies only target one specific condition, leading to high exclusion rates, especially in media-recruited samples. If participants with co-morbid disorders are included, the co-morbidity is generally not targeted, since the Internet treatment usually has manualised protocols leading to limited opportunities for participants and therapists to influence the treatment course. In face-to-face treatment, a behaviour analysis or a case conceptualisation is the starting point, and a treatment plan is designed around the information collected from the face-to-face appointment, by carrying out tailored treatments, these problems are addressed.

There is some evidence that tailored ICBT is a feasible option in a heterogeneous self-recruited group of anxiety participants [16]. In that study long-term treatment benefits were found. There is some additional evidence indicating that the effects of guided ICBT are maintained over longer time periods [17-19].

This study will consist of a sample referred via primary care contacts with the aim to test tailored ICBT in an effectiveness study.

\section{Methods/Design \\ Design}

We will conduct a randomised controlled trial with two conditions: (1) ICBT with scheduled guidance and (2) an active waiting list control group: "attention control". The study has been approved by the regional Ethical Board and has been registered in clinicaltrials.gov (NCT01390168).

\section{Study population}

The study population will consist of adult primary care patients fulfilling the DSM-IV-TR [20] criteria of any specific anxiety disorder, or anxiety disorder not otherwise specified. Hence, all participants must have clinically significant symptoms. Co-morbidity will not serve as a reason for exclusion, with the exception of health conditions that will hinder participation in the study and a few additional exclusion criteria. Participants will be included regardless of co-occurring depression; however, the anxiety disorder will have to be the primary diagnosis and complaint. Inclusion criteria will be the following: the person must be 18 years of age or older; they must have Internet access; they must be able to take part in a telephone-administered diagnosis interview; they should not be receiving any ongoing psychological treatment; and they should not be suffering from clinical or sub-clinical anxiety symptoms, if the patient is undergoing any medical treatment for their condition, the dosage has to be stable for at least three months prior to the start of the treatment. Participants with a suicidal risk, defined as scoring 4 or higher on item 9 of the Montgomery Åsberg Rating Scale-Self-rated (MADRS-S [21]), and who are judged to show a high risk in the SAD PERSONS [22] interview will be excluded. Participants with ongoing alcohol dependence will be excluded as well.

\section{Sample size}

Power calculations based on a moderate effect size of $d$ $=0.50$, comparing the Clinical Outcomes in Routine Evaluation-Outcome Measure (CORE-OM; [23]) scores of the intervention group with the control group with a two-sided t-test (alpha .05 , power $80 \%$ ). This is in accordance with Chambless and Hollon's [24] recommendations for empirically supported therapies. Hence, the $\mathrm{N}$ will be set at 128 participants, with 64 in each arm.

\section{Recruitment}

Participants will be recruited from a clinical populationtypically by their physician when seeking help at their primary care setting. Eligible participants will be given an information folder containing brief information about the trial and the link to the study website. On the website they will find further information, an informed consent form, a link to the questionnaires serving as screening and the application for participation. The informed consent form will be sent by surface post.

\section{Randomisation and procedure}

Initial selection will take place via computerised screening, consisting of the CORE-OM; [23], the self-rated version of the MADRS-S [25], Beck Anxiety Inventory (BAI;[26]), Quality of Life Inventory (QOLI;[27]), and 10 additional questions regarding demographics and current and past treatment. These results will later be used as a pretreatment assessment.

Participants who fulfil the initial inclusion criteria, according to the computerised screening, will be contacted to take part in a telephone interview where they will be interviewed using the Structured Clinical Interview for DSM-IV-Axis I Disorders (SCID-I; [28]) by seven MSc clinical psychology students trained in using the interview. A psychiatrist and a clinical psychologist will then assess the SCID protocols together with the 
interviewers. The interviewer, the psychiatrist and the clinical psychologist will subsequently tailor the treatment according to each participant's unique set of problems and to some extent preferences. All participants will be randomised by an online true random-number service independent of the investigators and therapists. The post-treatment assessment will take place 10 weeks after the participants have started their treatment, regardless of how many treatment modules have been completed. There will also be a measurement at 12 months post treatment. All assessments, except the diagnosis interview at pretreatment, are self-reported and will be conducted via the Internet.

All communication will be administered via an online messaging system resembling standard e-mailing systems connected to the study. All data is encrypted in the database and a cryptographic protocol (Secure Sockets Layer) will be used to provide communication security over the Internet.

\section{Intervention}

\section{Treatment}

The treatment will cover a period of 10 weeks and consist of a maximum of 10 modules, or chapters, per participant. The modules are based on cognitive-behavioural therapy (CBT) and shown to be effective when used on specific diagnosed samples (i.e. panic disorder [29], social phobia [30], GAD [19], depression [31]). Modules will be rewritten to fit together in the tailored format (e. g., words relating to specific conditions will be removed like "panic disorder").

Each module consists of a text (9-39 A4 pages) presenting a specific symptom (e.g. agoraphobia), exercises and is completed by three to eight essay questions to be worked through during a period of one week. Some of the modules (i.e. relaxation and mindfulness) have audio files attached to them for the participants to listen to. The homework questions are intended to encourage learning and to help the Internet therapist assess whether the participants have assimilated the material or not. Participants will be asked to answer the questions and provide worksheets and report on outcomes from different exercises to their therapist and will then be served with individual feedback. When the therapist receives a homework assignment showing that the participant has assimilated the material, the next module will be made accessible through an encrypted message exchange system. If not posted in time, the therapist will send a reminder message, and if the submitted homework does not show adequate knowledge, the therapist will send feedback to the participant regarding how to work on the area further. Prescribed modules are available for download in PDF format and participants will be advised to print out the self-help material to make it easier to take notes and to have the material readily available. The therapists will be told not to spend more than 15 minutes per participant per week in reading and communicating.

The first module (introduction) and the last (relapse prevention) are fixed, which gives the clinician the possibility to tailor the treatment by adding any of the following: cognitive restructuring ( 2 modules), social anxiety (2 modules), generalised anxiety (3 modules), panic disorder ( 2 modules), agoraphobia, behavioural activation (2 modules), applied relaxation, mindfulness, assertiveness, problem solving, stress management and sleep.

\section{Attention control}

Participants in the attention control group are asked questions about their well-being on a weekly basis by their therapist. Each participant will e-mail their answers to the therapist once a week, but they are generally not given any feedback on their answers.

\section{Instruments}

All questionnaires, except the diagnostic interview, will be administered via the Internet. The reason for doing this is to minimise the risk for keying errors and to be cost-effective. Research has shown that Internet-administered self-reporting of these questionnaires is as equally valid as the paper and pencil versions $[32,33]$. The diagnostic interview (SCID-I) will be conducted by telephone, a format shown to be as equally valid as faceto-face administration [34].

All instruments will be used at pretreatment, post treatment and at 12-month follow-up, with the exception of demographic data and diagnosis interview at post treatment and in the long-term follow-up.

\section{Demographic data}

Ten background questions will be asked about age, gender, education, past and previous treatment (both psychological and medication) and employment status.

\section{Diagnosis}

The Structured Clinical Interview for DSM-IV-Axis I disorders (SCID-I; [28]) will be used to investigate the presence of a diagnosis. This is a semi-structured interview to assess for DSM-IV Axis I-diagnoses.

\section{Primary outcomes}

The CORE-OM [23] is a 34-item scale covering four subscales of subjective well-being. The scales are: symptoms (anxiety, depression, physical problems, trauma), functioning (general functioning, close relationships, social relationships) and risk (to self and others). Items are scored from $0-4$ and relate to the preceding week. CORE-OM clinical scores can range from $0-40$, with higher scores indicating greater severity. Internal consistency has been reported as $\alpha=.94$ [23].

\section{Secondary outcomes}

For the measurement of anxiety symptoms, Beck Anxiety Inventory (BAI; [26]) will be used. The BAI contains 
21 short questions and scoring varies from 0-63. A score of 30 or higher is considered to correspond to severe anxiety symptoms. Internal consistency is high [26], with Cronbach's alpha ranging from .90 to .94 . and testretest reliability at $r=.75$.

Depressive symptoms will be measured using the MADRS-S [25]. The scale consists of nine items rated on a seven-grade scale measuring depressive symptoms over the past three days. MADRS-S was developed to measure changes in depressive symptoms and was originally a clinical-rated version. The self-rated version has shown a high degree of concordance with the clinical-rated one [35]. Total scoring varies from 0-54, with scores above 35 [36] considered as severe. This scale will also serve as an initial indicator for suicidal risk using the participants scoring on item 9 (suicidal thoughts) as a marker for further investigation of the matter. Concurrent validity is shown to be good with a test-retest reliability of $r=.83$ [37].

As noted earlier, the use of MADRS-S and the scoring on the suicidal thoughts item indicate the necessity for further investigation on suicidality via a telephone-administered interview conducted by a psychiatrist using the SAD PERSONS scale [22]. The SAD PERSONS is an acronym for 10 major risk factors (Sex, Age, Depression, Previous attempt, Ethanol abuse, Rational thinking loss, Social supports lacking, Organised plan, No spouse, Sickness) and is used together with a clinical evaluation carried out by the interviewer for assessing the likelihood of suicidal attempts [22].

For the measurement of quality of life we will use the QOLI [27]. This scale assesses subjective degree of satisfaction in 16 different areas of life (e.g. health and work). The scale show high internal consistency ( $\alpha=$ $.77-.89$ ), and a test-retest reliability from $r=.80$ to .91 [27].

For the measurement of loss of production and health-care consumption the Trimbos and Institute of Medical Technology Assessment Questionnaire on Costs Associated with Psychiatric Illness (TiC-P; [38]) will be used. We will use part I (direct costs of healthcare consumption) and part II (indirect costs of loss of production).

\section{Analyses}

When analysing post-treatment data and data collected at one-year follow-up, an intention-to-treat design will be used, to include all participants, regardless of their compliance, withdrawal or protocol deviations. Betweengroup changes at post treatment and at one-year-followup will be calculated using analyses of covariance (ANCOVAs), with pre-treatment scores as the covariate. This approach is proven to be a reliable strategy in analysing the results of baseline and follow-up data in
RCTs [39]. Effect sizes between and within the two groups will be calculated with Cohen's $d$ computed with the pooled standard deviation.

\section{Discussion}

The trial described in this protocol is a randomised controlled trial targeting individuals aged 18 years or older who seek their primary care setting for emotional distress. The individuals suitable for this treatment will be randomised into either treatment or an active control group. Outcome measures post treatment will be compared with those of the wait list control group. The primary aim is to assess the effectiveness of the tailored online treatment both post treatment and at a one-year follow-up.

Given the large number of individuals suffering from mental disorders, and the small proportion receiving adequate treatment for their condition, increasing the accessibility of effective treatment is important. Given that the bulk of patients fulfilling an anxiety diagnosis suffer from at least one co-morbid disorder [4] makes it important to investigate if the ICBT format of treatment is applicable to these individuals as well. The main part of earlier studies on ICBT has focused on only one primary diagnosis, leading to high exclusion rates of up to 84 per cent [29]. One of the aims of this study is to investigate whether this kind of treatment could be feasible for primary care patients regardless of anxiety diagnosis and co-morbidity, striving to test the effectiveness of this treatment.

The recruitment of participants to this study is designed to be similar to that of an ordinary process of seeking psychological treatment via a primary care setting. The patient and primary care contact will meet as usual, as they would if they were receiving treatment, and then the potential participant would be recommended to apply for the study. This leaves their primary care contact, in the first instance, to decide which treatment to recommend for the patient's unique set of symptoms. This increases the external validity.

This design has some limitations. One possibility is that the patients selected for the study will be considered being less severe patients-either by the characteristics of their symptoms or by demographic characteristics. Another limitation is the lack of control group in the one-year follow-up. For ethical reasons, the patients in our wait list will be offered the treatment after 10 weeks. In terms of generalisation, it should be taken into consideration that the sample could be selected on other criteria than they would be if they were receiving administered treatments. To reduce this risk, close contact between the primary care settings and the project leader of the study is strived for. Another fact is that the therapists in this study are 
students in later terms of clinical training, not a clinical psychologist or a counsellor that patients would normally meet in these settings. These issues are partly being addressed by the fact that the students undergo supervision from an experienced clinical psychologist.

\section{Trial status}

The trial is ongoing, still recruiting participants.

\section{Acknowledgements}

We thank the health-care providers in the primary care settings who are willing to recommend this novel treatment to their patients. This study is funded by the Swedish Council for Working Life and Social Research.

\section{Author details}

'Department of Behavioural Sciences and Learning, Linköping University, 581 83 Linköping, Sweden. ${ }^{2}$ Department of Clinical Neuroscience, Psychiatry Section, Karolinska Institutet, 17176 Stockholm, Sweden. ${ }^{3}$ Swedish Institute for Disability Research, Linköping University, 58183 Linköping, Sweden. ${ }^{4}$ County council of Östergötland, 58191 Linköping, Sweden. ${ }^{5}$ Department of Psychology, Umeå University, 90187 Umeå, Sweden.

\section{Authors' contributions}

LBN in collaboration with PC, GA and ÅK designed the study. LBN drafted the manuscript. PC and GA reviewed and revised the manuscript. All authors have read and approved the final manuscript to be published.

\section{Competing interests}

The authors declare that they have no competing interests.

Received: 22 November 2011 Accepted: 9 February 2012 Published: 9 February 2012

\section{References}

1. Smit F, Cuijpers P, Oostenbrink J, Batelaan N, de Graaf R, Beekman A: Costs of nine common mental disorders: Implications for curative and preventive psychiatry. Journal of Mental Health Policy and Economics 2006, 9:193-200.

2. Alonso J, Angermeyer MC, Bernert S, Bruffaerts R, Brugha TS, Bryson H, De Girolamo G, De Graaf R, Demyttenaere K, Gasquet D, et al: Prevalence of mental disorders in Europe: Results from the European Study of the Epidemiology of Mental Disorders (ESEMeD) project. Acta Psychiatrica Scandinavica, Supplement 2004, 109:21-27.

3. Wittchen HU, Jacobi F, Rehm J, Gustavsson A, Svensson M, Jönsson B, Olesen J, Allgulander C, Alonso J, Faravelli C, Fratiglioni L, Jennum P, Lieb R, Maercker A, van Os J, Preisig M, Salvador-Carulla L, Simon R, Steinhausen HC: The size and burden of mental disorders and other disorders of the brain in Europe 2010. European Neuropsychopharmacology 2011, 21:655-679.

4. Alonso J, Angermeyer MC, Bernert S, Bruffaerts R, Brugha TS, Bryson H, De Girolamo G, De Graaf R, Demyttenaere K, Gasquet I, et al: 12-Month comorbidity patterns and associated factors in Europe: Results from the European Study of the Epidemiology of Mental Disorders (ESEMeD) project. Acta Psychiatrica Scandinavica, Supplement 2004, 109:28-37.

5. Wittchen $\mathrm{HU}$, Jacobi F: Size and burden of mental disorders in Europe-A critical review and appraisal of 27 studies. European Neuropsychopharmacology 2005, 15:357-376.

6. The National Board of Health and Welfare: Nationella riktlinjer för vård vid depression och ångestsyndrom [National guidelines for depression and anxiety]. 2010

7. Andersson G, Bergström J, Buhrman M, Carlbring P, Holländare F, Kaldo V, Nilsson-Ihrfelt E, Paxling B, Ström L, Waara J: Development of a new approach to guided self-help via the Internet. The Swedish experience. Journal of Technology and Human Services 2008, 26:161-181.

8. Almlöv J, Carlbring P, Berger T, Cuijpers P, Andersson G: Therapist factors in internet-delivered cognitive behavioural therapy for major depressive disorder. Cognitive Behaviour Therapy 2009, 38:247-254.
9. Palmqvist B, Carlbring P, Andersson G: Internet-delivered treatments with or without therapist input: does the therapist factor have implications for efficacy and cost? Expert Review of Pharmacoeconomics \& Outcomes Research 2007, 7:291-297.

10. Spek V, Cuijpers P, Nyklicek I, Riper H, Keyzer J, Pop V: Internet-based cognitive behaviour therapy for symptoms of depression and anxiety: a meta-analysis. Psychological medicine 2007, 37:319-328.

11. Andersson G: Using the Internet to provide cognitive behaviour therapy. Behaviour Research and Therapy 2009, 47:175-180.

12. Reger MA, Gahm GA: A meta-analysis of the effects of Internet-and computer-based cognitive-behavioral treatments for anxiety. Journal of Clinical Psychology 2009, 65:53-75.

13. Andrews G, Cuijpers P, Craske MG, McEvoy P, Titov N: Computer therapy for the anxiety and depressive disorders is effective, acceptable and practical health care: A meta-analysis. PLOS ONE 2010, 5.

14. Cuijpers P, Marks IM, van Straten A, Cavanagh K, Gega L, Andersson G: Computer-aided psychotherapy for anxiety disorders: A meta-analytic review. Cognitive Behaviour Therapy 2009, 38:66-82.

15. Coull G, Morris PG: The clinical effectiveness of CBT-based guided selfhelp interventions for anxiety and depressive disorders: A systematic review. Psychological Medicine 2011, 41:2239-2252.

16. Titov N, Gibson M, Andrews G, McEvoy P: Internet treatment for social phobia reduces comorbidity. Australian and New Zealand Journal of Psychiatry 2009, 43:754-759.

17. Carlbring $P$, Maurin L, Törngren $C$, Linna E, Eriksson T, Sparthan E, Strååt $M$ Marquez von Hage C, Bergman-Nordgren L, Andersson G: Individuallytailored, Internet-based treatment for anxiety disorders: A randomized controlled trial. Behaviour Research and Therapy 2011, 49:18-24.

18. Carlbring P, Bergman Nordgren L, Furmark T, Andersson G: Long term outcome of Internet delivered cognitive-behavioural therapy for social anxiety disorder: A 30-month follow-up. Behav Res Ther 2009, 47:848-850

19. Paxling B, Almlöv J, Dahlin M, Carlbring P, Breitholtz E, Eriksson T, Andersson G: Guided Internet-delivered cognitive behavior therapy for generalized anxiety disorder: A randomized controlled trial. Cognitive Behaviour Therapy 2011, 40:159-173.

20. American Psychiatric Association: Diagnostic and statistical manual of mental disorders-Text Revision. 4 edition. Washington, DC, USA: American Psychiatric Press; 2000.

21. Montgomery SA, Asberg M: A new depression scale designed to be sensitive to change. British Journal of Psychiatry 1979, 134:382-389.

22. Patterson WM, Dohn HH, Bird J, Patterson GA: Evaluation of suicidal patients: the SAD PERSONS scale. Psychosomatics 1983, 24:343-345, 348.

23. Barkham M, Leach C, Lucock M, Evans C, Margison F, Mellor-Clark J, Benson L, Connell J, Audin K, McGrath G: Service profiling and outcomes benchmarking using the CORE-OM: Toward practice-based evidence in the psychological therapies. Journal of Consulting and Clinical Psychology 2001, 69:184-196.

24. Chambless DL, Hollon SD: Defining empirically supported therapies. Journal of Consulting and Clinical Psychology 1998, 66:7-18.

25. Svanborg P, Åsberg M: A new self-rating scale for depression and anxiety states based on the comprehensive psychopathological rating scale. ACTA Psychiatrica Scandinavica 1994, 89:21-28.

26. Beck AT, Epstein N, Brown G, Steer RA: An inventory for measuring clinical anxiety: Psychometric properties. Journal of Consulting and Clinical Psychology 1988, 56:893-897.

27. Frisch MB, Cornell J, Villanueva M, Retzlaff PJ: Clinical validation of the Quality of Life Inventory. A measure of life satisfaction for use in treatment planning and outcome assessment. Psychological Assessment 1992, 4:92-101.

28. First MB, Gibbon M, Spitzer RL, Williams JBW: Structured clinical interview for DSM-IV Axis I Disorders (SCID-I) Washington, D.C.: American Psychiatric PresS; 1997.

29. Carlbring P, Bohman S, Brunt S, Buhrman M, Westling BE, Ekselius $L$, Andersson G: Remote Treatment of Panic Disorder: A Randomized Trial of Internet-Based Cognitive Behavior Therapy Supplemented With Telephone Calls. American Journal of Psychiatry 2006, 163:2119-2125.

30. Andersson $G$, Carlbring P, Holmström A, Sparthan E, Furmark T, NilssonIhrfelt E, Buhrman M, Ekselius L: Internet-based self-help with therapist feedback and in vivo group exposure for social phobia: A randomized controlled trial. Journal of Consulting and Clinical Psychology 2006, 74:677-686. 
31. Vernmark K, Lenndin J, Bjärehed J, Carlsson M, Karlsson J, Öberg J, Carlbring P, Eriksson T, Andersson G: Internet administered guided selfhelp versus individualized e-mail therapy: A randomized trial of two versions of CBT for major depression. Behaviour Research and Therapy 2010, 48:368-376.

32. Carlbring P, Brunt S, Bohman S, Richards JC, Öst L-G, Andersson G: Internet vs. Paper and Pencil Administration of Questionnaires Commonly Used in Panic/Agoraphobia research. Computers in Human Behavior 2007, 23:1421-1434

33. Hedman E, Ljótsson B, Rück C, Furmark T, Carlbring P, Lindefors N, Andersson G: Internet administration of self-report measures commonly used in research on social anxiety disorder: A psychometric evaluation. Computers in Human Behavior 2010, 26:736-740.

34. Rohde P, Lewinsohn PM, Seeley JR: Comparability of telephone and faceto-face interviews in assessing axis I and II disorders. American Journal of Psychiatry 1997, 154:1593-1598.

35. Bondolfi G, Jermann F, Rouget BW, Gex-Fabry M, McQuillan A, DupontWillemin A, Aubry JM, Nguyen C: Self- and clinician-rated MontgomeryÅsberg Depression Rating Scale: Evaluation in clinical practice. Journal of Affective Disorders 2010, 121:268-272.

36. Snaith RP, Harrop FM, Newby DA, Teale C: Grade scores of the Montgomery-Asberg depression and the clinical anxiety scales. British Journal of Psychiatry 1986, 148:599-601.

37. Mattila-Evenden M, Svanborg P, Gustavsson P, Åsberg M: Determinants of self-rating and expert rating concordance in psychiatric out-patients, using the affective subscales of the CPRS. Acta Psychiatrica Scandinavica 1996, 94:386-396.

38. Hakkaart-Van Roijen L, Van Straten A, Donker M: Manual:Trimbos/iMTA Questionnaire for Costs Associated with Psychiatric Illness Rotterdam: Erasmus University; 2002.

39. Vickers AJ: Analysis of variance is easily misapplied in the analysis of randomized trials: A critique and discussion of alternative statistical approaches. Psychosomatic Medicine 2005, 67:652-655.

doi:10.1186/1745-6215-13-16

Cite this article as: Nordgren et al:: Tailored internet-administered treatment of anxiety disorders for primary care patients: study protocol for a randomised controlled trial. Trials 2012 13:16.

\section{Submit your next manuscript to BioMed Central and take full advantage of:}

- Convenient online submission

- Thorough peer review

- No space constraints or color figure charges

- Immediate publication on acceptance

- Inclusion in PubMed, CAS, Scopus and Google Scholar

- Research which is freely available for redistribution

Submit your manuscript at www.biomedcentral.com/submit 